\title{
Might as well face it, you're addicted to MYC
}

Deregulated expression of MYC enjoys an almost omnipresent status in tumours - hence its nickname, McGene. Wherever you look, there it is. So understanding how MYC propels and maintains tumour growth is paramount.

An important question is how MYC levels are regulated. Michael Cole and colleagues used affinity purification to identify MYC-

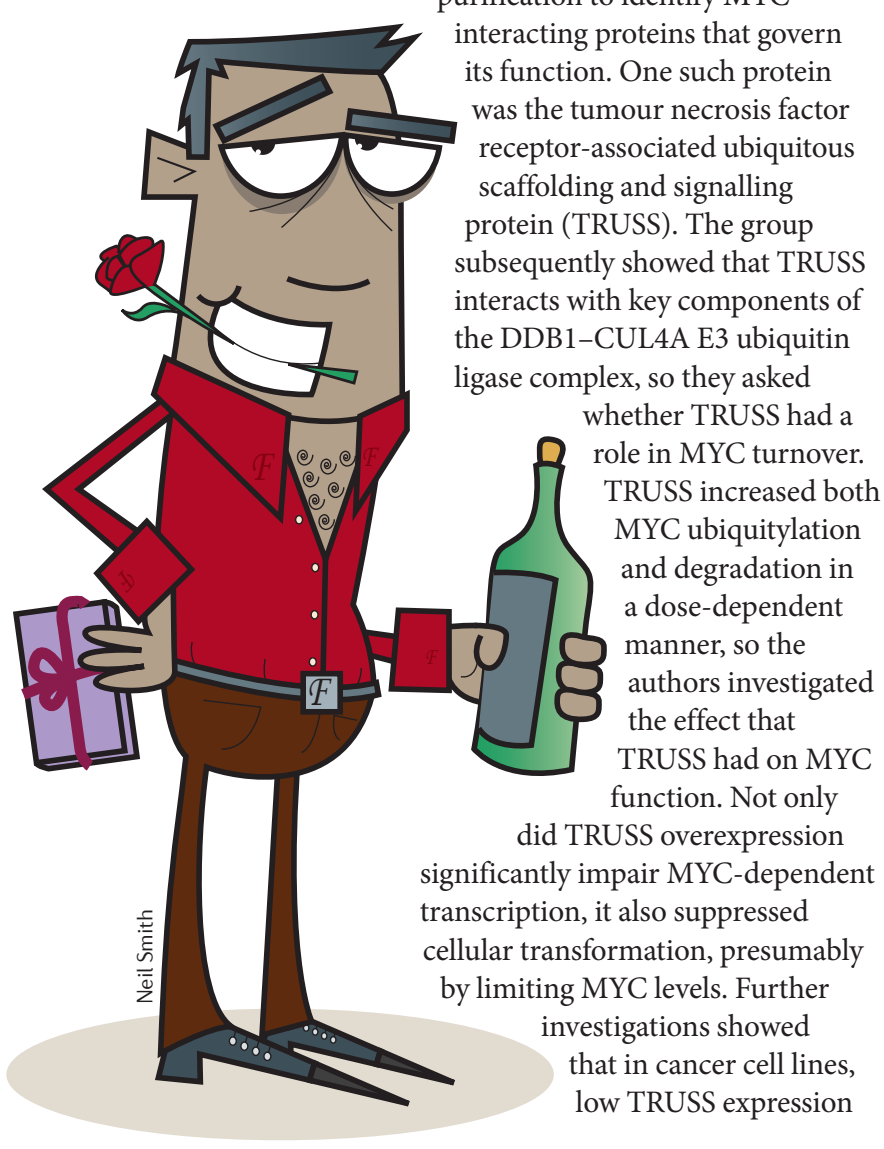

correlated with increased MYC protein half-life. Transformation is thought to be sensitive to MYC levels so the authors proposed that TRUSS might represent a critical barrier to MYC-dependent tumorigenesis.

Once released, how does MYC unleash its malignant potential? MYC, usually together with its partner MAX, activates a plethora of target genes that are involved in tumorigenesis, but the MYC-MAX heterodimer is also recruited to the transcription factor MIZ1, forming a repressive complex that abrogates MIZ1-dependent transcription. Is MYC-driven repression also required for tumour formation? Martin Eilers and Dean Felsher sought to find out.

Their groups teamed up and used a transcriptionally active mutant of MYC (MYCV394D) that cannot bind MIZ1 to ask how MIZ1 plays a part in T cell lymphomagenesis and why these tumours are addicted to MYC. The authors first noticed delayed lymphoma development in animals expressing MYCV394D, and staining for histone $\mathrm{H} 3$ trimethylation revealed high levels of senescence in these lymphomas, which was attributed to the observed increase in $C d k n 2 b$ (encoding INK4B) mRNA. Transforming growth factor- $\beta$ (TGF $\beta$ ) controls $C d k n 2 b$ expression in other cell types, and TGF $\beta$ levels were significantly higher in lymphomas than in normal or prelymphomagenic cells. Moreover, ectopic expression of TGF $\beta$ increased
$C d k n 2 b$ expression and induced senescence in cells expressing MYCV349D but not wild-type MYC. Therefore, lymphomagenesis activates an inherent, autocrine TGF $\beta$-dependent senescence programme that is short-circuited by MYC-mediated repression.

What about addiction to MYC? Eilers and Felsher generated lymphoma cells expressing MYC under a doxycycline-responsive promoter and either green fluorescent protein or the soluble TGF $\beta$ type II receptor extracellular domain (T $\beta$ R-II-ED), which abrogates TGF $\beta$ signalling. MYC expression was switched off once lymphomas had developed in syngeneic mice. Although initial tumour regression seems to be TGF $\beta$ independent, tumours rapidly recurred only in mice that were originally injected with T $\beta R$-II-ED-expressing cells. So, lowering MYC levels in lymphomas can trigger senescence, providing that the TGF $\beta$ autocrine pathway is functional. Therefore, if oncogenic addiction relies on stifling autocrine tumour-suppressive mechanisms, tumour regression might simply be a matter of restoring sensitivity by flipping the right switch.

Safia Ali Danovi

ORIGINAL RESEARCH PAPERS Choi, S. H. etal. Myc protein is stablilized by suppression of a novel E3 ligase complex in cancer cells. Genes Dev. 24, 1236-1241 (2010) | van Riggelen, J. et al. The interaction between Myc and Miz1 is required to antagonise TGF $\beta$-dependent autocrine signaling during lymphoma formation and maintenance. Genes Dev. 24, 1281-1294 (2010) 